\section{Hábitos de higiene bucal e utilização de serviços odontológicos em escolares de uma cidade da Região Sul do Brasil}

\author{
Oral hygiene habits and use of dental services \\ among teenage students in a city in southern \\ Brazil
}

\author{
1 Programa de Pós-gradu- \\ ação em Saúde Coletiva, \\ Universidade Luterana do \\ Brasil, Canoas, Brasil. \\ 2 Programa de Pós-graduação \\ em Odontologia, \\ Universidade Federal do \\ Rio Grande do Sul, \\ Porto Alegre, Brasil. \\ 3 Secretaria Municipal de \\ Educação de Gravataí, \\ Gravataí, Brasil. \\ Correspondência \\ S. L. Freddo \\ Programa de Pós-graduação \\ em Saúde Coletiva, \\ Universidade Luterana do \\ Brasil. \\ Rua Amélia Teles 167, \\ apto. 401, Porto Alegre, RS \\ 90460-070, Brasil. \\ freddente@ig.com.br
}

\begin{abstract}
This study evaluated oral hygiene habits and use of dental services among teenage students, and analyzed their association with sociodemographic factors and life styles. This cross-sectional study included a representative sample of 1,170 seventh-graders from municipal public schools of Gravataí, Rio Grande do Sul State, Brazil. The Cox regression model for univariate analysis, modified for cross-sectional studies, was used to analyze the association between variables. Of the adolescents included in the study, 77.8\% brushed their teeth three or more times a day, 31.9\% flossed daily, 68.9\% visited the dentist regularly, and $50 \%$ visited the dentist for dental treatment. Tooth brushing was more frequent among female adolescents. Lower socioeconomic status was associated with a lower frequency of daily flossing, fewer annual dental visits, and a greater prevalence of dental treatment visits. Similar results were found for adolescents with a sedentary lifestyle or that had tried smoking. The consumption of candy was associated with lower frequency of annual dental visits, and the consumption of soft drinks, with greater frequency of treatment visits. A healthy life style was associated with better oral hygiene habits and more frequent dental visits.
\end{abstract}

Oral Hygiene; Dental Health Services; Adolescents

\author{
Silvia Letícia Freddo 1 \\ Denise Rangel Ganzo de Castro Aerts 1 \\ Claídes Abegg 2 \\ Rosane Davoglio 1 \\ Patrícia Conzatti Vieira 1 \\ Lisiane Monteiro 3
}

\section{Introdução}

A adolescência é um período da vida importante para a formação do indivíduo e é nesta etapa que sua saúde geral encontra-se na melhor fase 1,2. Estudos têm demonstrado que hábitos de vida pouco saudáveis, durante a adolescência, constituem-se em fatores de risco para doenças, principalmente na vida adulta ${ }^{3}$. Esse também é um período de risco para a saúde bucal, pois as medidas adequadas de higiene podem entrar em conflito com o estilo de vida, já que nessa fase os adolescentes não mais aceitam a supervisão dos adultos. Em função disso, é necessário o desenvolvimento de medidas de educação em saúde na escola, por meio de programas que sejam capazes de atender às necessidades e às características próprias dos adolescentes 4,5,6,7.

Durante muito tempo, a cavidade bucal foi vista como uma estrutura anatômica isolada do resto do corpo. No entanto, ela está intimamente ligada ao indivíduo e, dependendo de suas condições, pode causar impacto positivo ou negativo sobre a saúde geral ${ }^{8}$. As práticas de higiene bucal, como a escovação dentária e o uso do fio dental, e o controle da dieta desempenham importante papel na prevenção das doenças bucais ${ }^{9}$. Em relação à freqüência diária de escovação dos dentes não existe consenso na literatura. Alguns autores recomendam uma freqüência de duas vezes ao dia 10,11, enquanto outros recomendam escovar três vezes ao dia 
e utilizar, diariamente, o fio dental 12,13,14, pois este é um instrumento eficaz para remoção da placa bacteriana interproximal e preservação da integridade periodontal 15.

Segundo alguns autores, a visita ao dentista deveria ser realizada uma vez ao ano para a manutenção de uma boa saúde bucal 11,16, sendo que o uso de serviços odontológicos, usualmente, é explicado por fatores epidemiológicos e sócio-demográficos, como raça 17,18 e condição social 19. Isso é, a utilização de serviços preventivos associa-se de forma positiva com a situação sócio-econômica, sendo que a falta de recursos financeiros é uma das mais importantes barreiras ao uso de serviços de saúde 20 .

Alguns autores argumentam que existe relação direta entre estilo de vida e hábitos de saúde bucal. Isso assume cada vez mais importância, pois, entre os jovens, os hábitos de risco à saúde bucal, como tabagismo, consumo de álcool e alimentação com produtos refinados e açucarados, são mais freqüentes 21,22,23,24,25,26,27,28.

Nesse contexto, o propósito principal do estudo foi investigar os hábitos de higiene bucal e a utilização de serviços odontológicos e sua associação com variáveis sócio-demográficas e relacionadas ao estilo de vida de escolares da rede pública municipal de Gravataí, Rio Grande do Sul, Brasil.

\section{Materiais e métodos}

O delineamento utilizado foi de estudo transversal, tendo como população alvo os 2.282 alunos matriculados nas sétimas séries das escolas públicas municipais de Ensino Fundamental de Gravataí, em março de 2005. Essa série foi escolhida em função do Ministério da Saúde tê-los eleito como grupo prioritário para a realização de ações de vigilância da saúde 29 .

O Município de Gravataí conta com uma população de 270.763 habitantes, sendo 91,2\% urbanos, e possui forte vocação industrial, com destaque ao pólo automotivo, setores do comércio e de serviços. A rede pública municipal de ensino é composta por 66 escolas que atendem cerca de 23 mil alunos, sendo a segunda maior do estado. São 52 escolas na zona urbana e 14 na zona rural, todas diurnas (Prefeitura Municipal de Gravataí. http://www.gravatai.rs.gov.br, acessado em 07/Jul/2007).

Para o cálculo da amostra, utilizou-se como parâmetros: nível de significância de 0,05, prevalência estimada de $50 \%$ para os desfechos de interesse e erro máximo tolerado de $\pm 3 \%$, produzindo uma amostra com 728 sujeitos. Com o objetivo de evitar um possível viés de deli- neamento, uma vez que foi utilizado um processo de amostragem que considerou a turma sorteada como conglomerado, foi utilizado um efeito de delineamento de 1,5 (364 sujeitos) e incluídos mais alunos, buscando compensar uma perda estimada em 20\% (220 alunos). Assim, a amostra final foi calculada em 1.312 escolares de sétima série. Como essa representava cerca da metade do total de alunos, optou-se por sortear metade mais uma das turmas existentes em cada uma das 15 regiões administrativas de Gravataí, garantindo a proporcionalidade de alunos em cada região. Com isso, o número de sujeitos selecionados foi de 1.366. As perdas ocorridas foram de 196 escolares (14,3\%), provindas de: transferência escolar (105), não autorização pelos pais ou responsáveis (30), recusas de alunos (31), expulsão (1), evasão (12), infreqüência (17), resultando uma amostra final de 1.170 escolares, provenientes de áreas urbanas e rurais.

Os dados foram coletados por profissionais e acadêmicos do setor saúde, previamente capacitados, utilizando dois questionários estruturados auto-aplicáveis e uma ficha coletiva. $\mathrm{O}$ primeiro questionário, desenvolvido para fins desta pesquisa, forneceu dados referentes ao estilo de vida sedentário; consumo de balas, refrigerantes e chocolates; sexo e inserção sócioeconômica. Para a caracterização da inserção sócio-econômica foi utilizada a classificação da Associação Brasileira de Empresas de Pesquisa (http://www.abep.org/codigosguias/ABEP_ CCEB.pdf, acessado em 10/Mar/2005). O segundo, elaborado pela Organização Mundial da Saúde (OMS) 30 para investigação da saúde do escolar, forneceu dados sobre o uso na vida (experiência) de tabaco e álcool. As questões sobre saúde bucal, incluídas nesse instrumento, foram adaptadas de outros estudos sobre o tema 31,32 . A ficha coletiva permitia o registro de cada aluno da turma, constando dados referentes à data de nascimento, cor da pele auto-referida e outros dados utilizados com fins administrativos para o controle do estudo.

Os desfechos de saúde bucal investigados na análise descritiva foram: freqüência diária de escovação dentária (uma, duas, três ou mais vezes); uso diário de fio dental (sim, não e às vezes); freqüência de utilização de serviços odontológicos (seis em seis meses, anual, dois em dois anos, somente quando há dor e não utiliza); e motivo da utilização do serviço (tratamento curativo, tratamento preventivo e não utiliza). Para fins das análises univariadas, foram considerados como desfechos: (1) freqüência não adequada de escovação dentária, quando inferior a três vezes ao dia (sim/não) 12,13,14; (2) uso não adequado de fio dental, quando não utili- 
zado diariamente (sim/não) 33,34. Nessa última variável, as categorias "não" e "às vezes” foram agrupadas, em função de não se caracterizarem como uso diário; (3) freqüência não adequada de utilização de serviços odontológicos, quando superior a uma vez ao ano (sim/não) 16; e (4) motivo da utilização do serviço para tratamento curativo (sim/não).

Neste estudo, considerou-se como adequada uma freqüência de escovação dentária de, no mínimo, três vezes ao dia, por se tratar de uma população de adolescentes, faixa etária em maior vulnerabilidade para doenças da cavidade bucal 4, na qual as medidas de auto cuidado e os comportamentos protetores são mais negligenciados.

As variáveis indicadoras da dimensão demográfica e sócio-econômica foram: sexo, cor da pele auto-referida (branca e não-branca) e inserção sócio-econômica. Não houve nenhum escolar pertencente à categoria $\mathrm{A}$ e os das categorias $\mathrm{D}$ e E foram agrupados, em função do pequeno número de jovens (6 casos) classificados na categoria E. Assim, a classificação sócio-econômica foi formada pelas categorias B, C e D+E.

As indicadoras do estilo de vida do escolar foram: sedentarismo (sim e não); uso na vida de álcool e tabaco (sim e não); consumo de balas, refrigerantes e chocolates (sim e não). Foi considerado como tendo estilo de vida sedentário um jovem que tenha referido a prática de esportes ou exercício físico com freqüência inferior a uma vez na semana e que não se deslocava para a escola a pé ou de bicicleta.

Para medir a associação entre os desfechos e os fatores em estudo, foi utilizada a regressão de Cox univariada, modificada para estudos transversais 35 , considerando o tempo como uma constante. Para tanto, foi utilizado o programa Stata versão 6.0 (Stata Corp., College Station, Estados Unidos). Com isso, foi possível o cálculo das razões de prevalência (prevalência entre expostos/prevalência entre não-expostos), intervalos de confiança, sendo considerado estatisticamente significativo um valor de $\mathrm{p}<0,05$.

Antes do início da pesquisa, fez-se necessário um contato com os responsáveis pelas escolas, para explicar os objetivos da mesma e a forma como esta seria conduzida. Em reunião com os pais ou responsáveis pelos alunos foi obtido o Termo de Consentimento Livre e Esclarecido. Esta pesquisa faz parte de um estudo maior intitulado A Saúde do Escolar da Rede Pública Municipal de Gravataí-RS, que foi aprovada pelo Comitê de Ética da Universidade Luterana do Brasil (ULBRA -2004-375H).

\section{Resultados}

Dos escolares investigados, 556 (47,5\%) eram do sexo masculino; 615 (52,6\%) se auto-referiram como tendo a cor da pele branca; 226 (19,3\%) pertenciam à categoria sócio-econômica mais baixa (D+E); 688 (58,8\%) à C; $256(21,9 \%)$ à $\mathrm{B}$, sendo que nenhum escolar foi classificado na categoria A. A média de idade foi de 14 anos (DP = 1,13 anos).

Considerou-se que a maioria dos escolares apresentava uma freqüência de escovação adequada, pois $77,8 \%$ escovavam os dentes três ou mais vezes ao dia. Todavia, apenas $31,9 \%$ faziam uso diário de fio dental. Quanto à freqüência de utilização de serviços odontológicos, $68,9 \%$ visitaram o dentista pelo menos uma vez ao ano e $41,1 \%$ buscaram atendimento para tratamento curativo, como dor de dente, acidente, traumatismo bucal, cárie, sangramento gengival, refazer tratamento e 8,9\% não consultaram (Tabela 1).

Em relação à freqüência de escovação dentária, entre todas as variáveis estudadas, somente o sexo associou-se de forma significati-

Tabela 1

Distribuição dos escolares segundo hábitos de higiene bucal e utilização de serviços odontológicos. Gravataí, Rio Grande do Sul, Brasil, 2005.

\begin{tabular}{|c|c|c|}
\hline Variáveis & $\mathbf{n}$ & $\%$ \\
\hline \multicolumn{3}{|c|}{ Freqüência diária de escovação } \\
\hline$\leq 1 \mathrm{vez}$ & 45 & 3,8 \\
\hline 2 vezes & 214 & 18,3 \\
\hline$\geq 3$ vezes & 911 & 77,9 \\
\hline \multicolumn{3}{|l|}{ Uso diário de fio dental } \\
\hline $\operatorname{Sim}$ & 373 & 31,9 \\
\hline Não & 391 & 33,4 \\
\hline Às vezes & 406 & 34,7 \\
\hline \multicolumn{3}{|c|}{ Freqüência de utilização de serviços } \\
\hline 6 em 6 meses & 547 & 46,8 \\
\hline 1 vez ao ano & 259 & 22,1 \\
\hline 2 em 2 anos & 27 & 2,3 \\
\hline Somente quando há dor & 159 & 13,6 \\
\hline Não utiliza & 178 & 15,2 \\
\hline \multicolumn{3}{|l|}{ Tipo de serviço utilizado } \\
\hline Não privado & 787 & 67,3 \\
\hline Privado & 261 & 22,3 \\
\hline Não utiliza & 122 & 10,4 \\
\hline \multicolumn{3}{|c|}{ Motivo da utilização do serviço } \\
\hline Curativo & 586 & 50,0 \\
\hline Preventivo & 480 & 41,1 \\
\hline Não utiliza & 104 & 8,9 \\
\hline Total & 1.170 & 100,0 \\
\hline
\end{tabular}


va, mostrando que os meninos têm $80 \%$ mais freqüência não adequada do que as meninas. Apesar desse desfecho ser mais freqüente entre escolares de cor da pele branca, pertencentes à inserção sócio-econômica mais baixa $(\mathrm{D}+\mathrm{E})$, os sedentários, que já experimentaram bebidas alcoólicas e não consumidores de refrigerantes ou chocolates, estas diferenças não foram significativas. Da mesma forma, não se evidenciou associação entre a freqüência de escovação e o consumo de balas (Tabela 2).

O uso não adequado de fio dental foi $19 \%$ maior entre os adolescentes da categoria sócioeconômica D+E, $23 \%$ maior entre os sedentários e $10 \%$ maior entre os que já experimentaram cigarro. Por outro lado, os jovens consumidores de chocolates tiveram menor freqüência (10\%) de uso não adequado de fio dental. Isso é, o consumo de chocolates esteve associado a uma maior prevalência desse hábito. As demais variáveis não se associaram a esse desfecho (Tabela 2).

Quanto à utilização de serviços odontológicos, não houve associação com as variáveis: sexo, cor da pele, uso de álcool e consumo de refrigerantes e chocolates. Os escolares pertencentes às categorias sócio-econômicas D+E e C utilizaram os serviços odontológicos com menor freqüência que os da $\mathrm{B}$, sendo estas diferenças estatisticamente significativas. Entre os adolescentes com estilo de vida sedentário, a freqüência não adequada de utilização foi $70 \%$ maior. Da mesma forma, também se encontrou associação entre esse desfecho e o uso de tabaco e consumo de balas. Os adolescentes que experimentaram cigarro e os que consumiam balas

Fatores associados à freqüência não adequada de escovação e uso não adequado de fio dental. Gravataí, Rio Grande do Sul, Brasil, 2005.

\begin{tabular}{|c|c|c|c|c|c|c|c|c|c|c|c|c|}
\hline & \multirow[t]{2}{*}{$\mathbf{n}$} & \multirow[t]{2}{*}{$\%$} & \multicolumn{5}{|c|}{ Freqüência não adequada de escovação dentária } & \multicolumn{5}{|c|}{ Uso não adequado de fio dental } \\
\hline & & & $n$ & $\%$ & RP & IC95\% & $p$ & $\mathbf{n}$ & $\%$ & RP & IC95\% & $\mathrm{p}$ \\
\hline \multicolumn{13}{|l|}{ Sexo } \\
\hline Masculino & 556 & 47,5 & 161 & 29,0 & 1,80 & $1,44-2,24$ & 0,000 & 389 & 70,0 & 1,05 & $0,97-1,14$ & 0,197 \\
\hline Feminino & 614 & 52,5 & 99 & 16,1 & 1,00 & - & - & 408 & 66,4 & 1,00 & - & - \\
\hline \multicolumn{13}{|l|}{ Cor da pele } \\
\hline Branca & 615 & 52,6 & 143 & 23,3 & 1,00 & - & - & 413 & 67,1 & 1,00 & - & - \\
\hline Não-branca & 555 & 47,4 & 117 & 21,1 & 0,90 & $0,73-1,12$ & 0,373 & 384 & 69,2 & 1,03 & $0,95-1,11$ & 0,455 \\
\hline \multicolumn{13}{|l|}{ Classificação } \\
\hline \multicolumn{13}{|c|}{ sócio-econômica } \\
\hline B & 256 & 21,9 & 57 & 22,3 & 1,00 & - & - & 162 & 63,2 & 1,00 & - & - \\
\hline $\mathrm{C}$ & 688 & 58,8 & 147 & 21,4 & 0,96 & $0,73-1,26$ & 0,765 & 465 & 67,6 & 1,06 & $0,95-1,18$ & 0,237 \\
\hline$D+E$ & 226 & 19,3 & 56 & 24,8 & 1,11 & $0,81-1,54$ & 0,516 & 171 & 75,7 & 1,19 & $1,06-1,34$ & 0,003 \\
\hline \multicolumn{13}{|l|}{ Sedentarismo } \\
\hline Não & 1.034 & 88,4 & 224 & 21,7 & 1,00 & - & - & 687 & 66,4 & 1,00 & - & - \\
\hline Sim & 135 & 11,6 & 36 & 26,7 & 1,23 & $0,91-1,67$ & 0,179 & 110 & 81,4 & 1,23 & $1,12-1,34$ & 0,000 \\
\hline \multicolumn{13}{|l|}{ Uso de álcool } \\
\hline Sim & 710 & 60,7 & 162 & 22,9 & 1,07 & $0,86-1,34$ & 0,544 & 487 & 69,7 & 1,05 & $0,96-1,14$ & 0,235 \\
\hline Não & 460 & 39,3 & 98 & 21,3 & 1,00 & - & - & 310 & 65,7 & 1,00 & - & - \\
\hline \multicolumn{13}{|l|}{ Uso de tabaco } \\
\hline Sim & 195 & 16,7 & 44 & 22,6 & 1,02 & $0,77-1,35$ & 0,899 & 144 & 73,8 & 1,10 & $1,01-1,21$ & 0,043 \\
\hline Não & 975 & 83,3 & 216 & 22,1 & 1,00 & - & - & 653 & 67,0 & 1,00 & - & - \\
\hline \multicolumn{13}{|c|}{ Consumo de balas } \\
\hline Sim & 825 & 70,5 & 78 & 22,6 & 0,97 & $0,77-1,23$ & 0,837 & 568 & 68,8 & 1,04 & $0,95-1,13$ & 0,416 \\
\hline Não & 345 & 29,5 & 182 & 22,7 & 1,00 & - & - & 229 & 66,3 & 1,00 & - & - \\
\hline \multicolumn{13}{|c|}{ Consumo de refrigerantes } \\
\hline Sim & 950 & 81,2 & 202 & 21,2 & 0,81 & $0,63-1,04$ & 0,095 & 641 & 67,4 & 0,95 & $0,86-1,05$ & 0,308 \\
\hline Não & 220 & 18,8 & 58 & 26,3 & 1,00 & - & - & 156 & 71,0 & 1,00 & - & - \\
\hline \multicolumn{13}{|c|}{ Consumo de chocolates } \\
\hline Sim & 596 & 50,9 & 124 & 20,8 & 1,13 & $0,91-1,41$ & 0,236 & 387 & 64,9 & 0,90 & $0,84-0,98$ & 0,017 \\
\hline Não & 574 & 49,1 & 136 & 23,7 & 1,00 & - & - & 410 & 71,4 & 1,00 & - & - \\
\hline
\end{tabular}


apresentaram uma freqüência não adequada de utilização de serviços odontológicos, cerca de, $30 \%$ maior do que seus pares de referência (Tabela 3).

Em relação ao motivo pelo qual os adolescentes procuravam o serviço, comparados aos da categoria sócio-econômica $\mathrm{B}$, os da C tiveram uma prevalência $19 \%$ maior de procura por tratamento curativo e os da categoria $\mathrm{D}+\mathrm{E}$, $51 \%$. Os com estilo de vida sedentário, os que experimentaram cigarro e os que consumiam refrigerantes também tiveram mais consultas curativas do que seus pares de referência. Em contrapartida, as variáveis sexo, cor da pele, uso de álcool, consumo de balas e chocolates não se associaram a esse desfecho (Tabela 3 ).

\section{Discussão}

O presente estudo foi realizado com uma amostra representativa dos adolescentes de sétima série das escolas públicas municipais de Gravataí, pois foram selecionados em todas as 15 regiões do município, sendo investigado um número superior ao tamanho mínimo de amostra calculada. Acredita-se também que possa ser representativa dos escolares da Região Metropolitana de Porto Alegre, uma vez que as cidades que compõem esta região apresentam características sócio-demográficas semelhantes. No entanto, como a pesquisa foi realizada em escolas, os resultados não podem ser extrapolados para a população de adolescentes não escolares, pois os jovens que freqüentam a escola

Fatores associados à freqüência não adequada de consultas odontológicas e ao motivo curativo da consulta. Gravataí, Rio Grande do Sul, Brasil, 2005.

\begin{tabular}{|c|c|c|c|c|c|c|c|c|c|c|c|c|}
\hline & \multirow[t]{2}{*}{$\mathbf{n}$} & \multirow[t]{2}{*}{$\%$} & \multicolumn{5}{|c|}{ Freqüência não adequada de consultas } & \multicolumn{5}{|c|}{ Motivo curativo } \\
\hline & & & $\mathrm{n}$ & $\%$ & RP & IC95\% & $\mathrm{p}$ & $\mathbf{n}$ & $\%$ & $\mathrm{RP}$ & IC95\% & $\mathrm{p}$ \\
\hline \multicolumn{13}{|l|}{ Sexo } \\
\hline Masculino & 556 & 47,5 & 180 & 32,3 & 1,08 & $0,91-1,28$ & 0,375 & 275 & 49,5 & 1,00 & $0,90-1,17$ & 0,986 \\
\hline Feminino & 614 & 52,5 & 184 & 30,0 & 1,00 & - & - & 311 & 50,6 & 1,00 & - & - \\
\hline \multicolumn{13}{|l|}{ Cor da pele } \\
\hline Branca & 615 & 52,6 & 181 & 29,4 & 1,00 & - & - & 301 & 48,9 & 1,00 & - & - \\
\hline Não-branca & 555 & 47,4 & 183 & 33,0 & 1,12 & $0,94-1,32$ & 0,191 & 287 & 51,3 & 1,05 & $0,94-1,17$ & 0,362 \\
\hline \multicolumn{13}{|l|}{ Classificação } \\
\hline \multicolumn{13}{|c|}{ sócio-econômica } \\
\hline B & 256 & 21,9 & 55 & 21,4 & 1,00 & - & - & 111 & 43,3 & 1,00 & - & - \\
\hline C & 688 & 58,8 & 147 & 21,3 & 1,38 & $1,06-1,80$ & 0,014 & 341 & 49,5 & 1,19 & $1,02-1,39$ & 0,025 \\
\hline$D+E$ & 226 & 19,3 & 56 & 24,8 & 2,14 & $1,62-2,81$ & 0,000 & 134 & 59,3 & 1,51 & $1,28-1,79$ & 0,000 \\
\hline \multicolumn{13}{|l|}{ Sedentarismo } \\
\hline Não & 1.034 & 88,4 & 298 & 28,8 & 1,00 & - & - & 501 & 58,4 & 1,00 & - & - \\
\hline Sim & 135 & 11,6 & 66 & 48,9 & 1,70 & $1,39-2,06$ & 0,000 & 85 & 63,0 & 1,32 & $1,16-1,51$ & 0,000 \\
\hline \multicolumn{13}{|l|}{ Uso de álcool } \\
\hline Sim & 698 & 59,7 & 220 & 31,5 & 1,02 & $0,86-1,22$ & 0,785 & 353 & 50,5 & 1,02 & $0,91-1,14$ & 0,717 \\
\hline Não & 472 & 40,3 & 144 & 30,5 & 1,00 & - & - & 233 & 49,3 & 1,00 & - & - \\
\hline \multicolumn{13}{|l|}{ Uso de tabaco } \\
\hline $\operatorname{Sim}$ & 195 & 16,7 & 76 & 39,0 & 1,32 & $1,08-1,61$ & 0,007 & 113 & 57,8 & 1,20 & $1,06-1,36$ & 0,005 \\
\hline Não & 975 & 83,3 & 288 & 29,5 & 1,00 & - & - & 473 & 48,5 & 1,00 & - & - \\
\hline \multicolumn{13}{|c|}{ Consumo de balas } \\
\hline Sim & 825 & 70,5 & 276 & 33,4 & 1,31 & $1,07-1,61$ & 0,009 & 423 & 51,2 & 1,09 & $0,97-1,24$ & 0,139 \\
\hline Não & 345 & 29,5 & 88 & 25,5 & 1,00 & - & - & 163 & 47,2 & 1,00 & - & - \\
\hline \multicolumn{13}{|c|}{ Consumo de refrigerantes } \\
\hline Sim & 950 & 81,1 & 302 & 31,8 & 1,13 & $0,89-1,42$ & 0,306 & 495 & 56,8 & 1,22 & $1,04-1,43$ & 0,016 \\
\hline Não & 220 & 18,9 & 62 & 28,1 & 1,00 & - & - & 91 & 41,3 & 1,00 & - & - \\
\hline \multicolumn{13}{|c|}{ Consumo de chocolates } \\
\hline Sim & 596 & 50,9 & 182 & 30,5 & 0,96 & $0,81-1,14$ & 0,666 & 288 & 48,3 & 0,94 & $0,85-1,05$ & 0,307 \\
\hline Não & 574 & 49,1 & 182 & 31,7 & 1,00 & - & - & 298 & 51,9 & 1,00 & - & - \\
\hline
\end{tabular}


têm mais chance de receber orientação e de desenvolverem hábitos saudáveis 6,7 .

Em função do delineamento utilizado, é importante ressaltar que as associações encontradas indicam a concomitância das situações investigadas, não podendo ser interpretadas como relações do tipo causa e efeito.

O padrão de escovação diária dos dentes foi alto, sendo que a maior parte dos jovens declarou escovar três ou mais vezes ao dia. Estudos realizados nos Estados Unidos e na Europa demonstram que, nestes países, a freqüência de escovação mais comum é uma ou duas vezes ao dia 36 . No Brasil, o padrão é mais alto. Os resultados encontrados são similares aos descritos por Brew 37 , Abegg 38 e Lisbôa 31, no Rio Grande do Sul, onde a maioria dos adolescentes entrevistados escovava os dentes três ou mais vezes ao dia, apesar não utilizarem fio dental regularmente.

Diferentemente desses autores, Flores \& Drehmer 39, em Porto Alegre, Rio Grande do Sul, evidenciaram que $66 \%$ dos escolares utilizam fio dental diariamente, assim como Rodrigues 40 , em adolescentes atletas de São Paulo. Nesta pesquisa, o uso diário de fio dental foi inferior a um terço. Resultado semelhante ao verificado em outro estudo 37 , apesar da grande variedade de fios disponível no mercado e da recomendação de seu uso para complementar a higiene bucal.

Na investigação da relação entre os desfechos indicadores da higiene bucal e os fatores em estudo, encontrou-se associação entre freqüência de escovação dentária inferior a três vezes ao dia e sexo masculino. Esse resultado é confirmado por outros autores $38,41,42$, que observaram uma menor freqüência de escovação entre os homens. Dentre as explicações para esse comportamento estaria a de que os hábitos preventivos são mais comuns entre as mulheres, principalmente por questões estéticas ou normas sociais 38,43,44.

Nesta pesquisa, não se encontrou associação entre inserção sócio-econômica e freqüência de escovação, diferentemente de estudos realizados na China 19, Finlândia 21, Estados Unidos 17 e na cidade de São Paulo 18 que encontraram relação direta com nível sócio-econômico. É possível que não se tenha encontrado associação devido a uma maior homogeneidade da amostra, já que os alunos se concentraram nas categorias sócio-econômicas intermediárias, não existindo nenhum pertencente à categoria A e apenas seis na E, determinando que estes fossem agrupados com os da categoria D. No entanto, parece que o fator que mais contribui para a diminuição da influência das condições sócio-econômicas sobre a escovação foi que a Prefeitura Municipal de Gravataí distribui escovas de dentes gratuitamente aos escolares de sua rede de ensino. Com isso, houve uma redução da importância do fator econômico na compra do produto, além de contribuir, positivamente, para a incorporação do seu uso nos hábitos de higiene dos jovens, independente dos hábitos da família. Talvez, essa também seja uma possível explicação para a não associação entre as variáveis indicadoras da dieta cariogênica (consumo de balas, refrigerantes e chocolates). Entretanto, também é possível que a forma como essas variáveis foram coletadas, perguntando-se ao escolar se ele habitualmente consumia esses produtos, sem medir a freqüência precisa de seu consumo, tenha contribuído para a não identificação de associações.

No que diz respeito ao uso de fio dental, escolares pertencentes à categoria $\mathrm{D}+\mathrm{E}$ apresentaram maior prevalência de uso não adequado. Nesse caso, parece que os de menor poder aquisitivo fazem menos uso do produto, talvez por desinformação ou mesmo por maior dificuldade para sua compra. Em comparação com a escova de dentes, o fio dental é um produto mais caro e de uso não tão difundido, sendo ainda pouco incorporado no cotidiano da população mais carente. Além disso, diferentemente das escovas dentárias, não é distribuído para os alunos nas escolas. Estudo realizado na Finlândia, também mostrou associação entre alto nível social e hábitos de limpeza interdental 21.

Em uma amostra de jovens atletas, foi identificada uma freqüência de uso diário de fio dental de $50 \%$. No presente estudo, o estilo de vida sedentário foi considerado como um indicador de hábitos poucos saudáveis. Em contrapartida, os escolares não-sedentários, os que tinham bons hábitos de higiene bucal e os que não haviam experimentado álcool e tabaco foram considerados adeptos de um estilo de vida saudável. Entre os escolares sedentários, a freqüência de uso não adequado do fio dental foi maior.

Também foi encontrada associação entre consumo de chocolates e menor prevalência de uso não adequado de fio dental. Isso é, os escolares que consomem chocolates informaram um maior uso diário de fio dental do que os que não consumiam. Esse resultado chama atenção, já que o mesmo não foi encontrado em relação ao consumo de balas ou refrigerantes. Inicialmente, acreditou-se haver relação entre consumo de chocolates e melhor inserção sócio-econômica, em função do custo deste produto, porém as análises complementares realizadas não confirmaram esta hipótese. Assim, é possível que o consumo de chocolates não esteja diretamente relacionado às condições econômicas da família do escolar e, sim, a um conjunto de práticas e hábitos mais saudáveis de vida, pois, parte dos 
jovens que consumia este produto, não consumia balas ou refrigerantes.

A freqüência de utilização de serviços odontológicos tem sido pouco investigada entre adolescentes escolares no Brasil. A recomendação usual em relação à periodicidade de visita ao dentista é de uma consulta anual, aumentando a possibilidade da identificação precoce de problemas de saúde bucal, em especial entre crianças e adolescentes 11,16. Ao mesmo tempo, a contribuição dos serviços na melhoria da saúde bucal, ainda que significativa, é considerada menor do que a das melhorias nas condições de vida da população 45 .

Estudo realizado com 344.875 indivíduos de várias faixas etárias, em nove regiões metropolitanas brasileiras, observou que os escolares foram os que mais visitaram o dentista, com freqüência entre $40 \%$ e $48 \%$. Também constatou que as categorias sócio-econômicas mais altas apresentaram menor número de indivíduos que nunca foram ao dentista 46,47 .

Neste trabalho, encontrou-se associação entre freqüência de utilização de serviços odontológicos e inserção sócio-econômica, sendo que, entre os mais carentes, as consultas foram realizadas com intervalo de tempo superior a $12 \mathrm{me}$ ses. A Pesquisa Nacional por Amostra de Domicílios, de 1998, evidenciou que há baixa utilização de serviços odontológicos e grandes diferenciais entre os grupos de maior e menor renda ${ }^{3}$. Resultado esse reforçado por estudos desenvolvidos recentemente na cidade de Bambuí, Minas Gerais, que verificaram que maior escolaridade e renda estavam positivamente associadas à visita regular ao dentista, no último ano, independente do sexo e da faixa etária 48,49 .

Também houve associação entre estilo de vida sedentário e esse desfecho, mostrando que esses adolescentes procuraram menos o dentista, assim como os que experimentaram cigarros e consumiam balas. Conforme mencionado anteriormente, essas variáveis foram utilizadas como indicadoras da presença ou ausência de hábitos saudáveis. Assim, é compreensível que os jovens que não apresentavam um estilo de vida saudável tivessem uma menor prevalência de consultas odontológicas, em especial para revisões periódicas.

Pinheiro et al. 16 observaram que a utilização de serviços odontológicos varia entre os estados: a melhor situação foi encontrada em estados do Sul e as piores nos do Norte e Nordeste. Segundo os autores, diferenças regionais, relacionadas a variações na distribuição geográfica; na quantidade de oferta de serviços odontológicos e programas de atenção à saúde bucal; no estímulo ao uso de serviços preventivos, na percepção do estado de saúde; e nos comportamentos e hábitos ligados à saúde bucal, podem explicar as diferenças na utilização de serviços odontológicos entre os estados.

O presente estudo não encontrou associação entre sexo e freqüência de utilização de serviços odontológicos, semelhante aos resultados encontrados por outro estudo brasileiro na faixa etária entre 10 e 14 anos 47. Diferentemente, outros autores 21,46,50 investigando a população em geral, evidenciaram que as mulheres procuravam o serviço com maior freqüência. As razões para as visitas mais freqüentes de mulheres têm sido relacionadas a questões estéticas, maior sensibilidade à dor e ao desconforto e necessidade de procurar auxílio ${ }^{49}$. No entanto, é possível que, para os jovens na faixa etária estudada, em função de ainda serem dependentes de cuidados dos adultos para a marcação de consultas, a influência do sexo não seja verificada.

Em relação ao motivo da visita ao serviço odontológico, não se identificou diferenças entre os sexos. No entanto, evidenciou-se a influência da situação sócio-econômica na razão pela busca de atendimento curativo, semelhante a estudo realizado na África do Sul 51 que observou que a condição sócio-econômica influenciava mais fortemente o tipo de tratamento odontológico recebido do que a necessidade clínica. Lisbôa \& Abegg 31 constataram que a busca de tratamento curativo foi relatada por mais de $60 \%$ dos jovens, resultado semelhante ao verificado em Gravataí.

Nos Estados Unidos, a presença de cáries e restaurações esteve inversamente associada com o nível sócio-econômico das comunidades estudadas 52 . Esse quadro de desigualdades no tipo de tratamento oferecido é reforçado pelos resultados do estudo realizado em Minas Gerais, comparando serviços privados, públicos e de sindicatos. Os autores observaram que os serviços públicos tendiam a fazer muito mais tratamentos curativos 48. Para Watt \& Sheiham 53, a redução das doenças bucais deve-se, em maior parte, ao uso da pasta de dente fluoretada e às melhorias nas condições sócio-econômicas, enquanto os serviços especializados, sejam curativos ou preventivos, não tiveram grande impacto.

Também se evidenciou associação entre visita ao serviço para tratamento curativo e estilo de vida sedentário, experiência de uso de tabaco e consumo de refrigerantes. Não foram encontrados na literatura estudos que investigassem essas associações em escolares. Entretanto, considerando essas variáveis como indicadoras de estilo de vida, é possível pensar que se o adolescente não está inserido em um contexto que favoreça a adoção de hábitos saudáveis, não buscará ser- 
viços odontológicos periodicamente, utilizandoos apenas em situações de doença.

\section{Considerações finais}

A alta prevalência de escovação adequada, possivelmente, deve-se à distribuição gratuita da escova, o que não se verificou em relação ao fio dental, medida complementar à escovação. Sugere-se que essa iniciativa seja adotada por outros municípios, pois a escola é um local privilegiado para a promoção da saúde geral e bucal dos jovens 4,7 .

As variáveis indicadoras do estilo de vida, como o consumo de produtos cariogênicos, de tabaco e sedentarismo, estiveram associadas aos desfechos estudados, mostrando que os hábitos de higiene bucal e a utilização de serviços odontológicos fazem parte de um conjunto maior de hábitos e comportamentos relacionados à boa saúde geral. Nesse sentido, a participação do educador junto ao escolar vem reforçar ou,

\section{Resumo}

Estudo transversal investigando associação entre hábitos de higiene bucal e utilização dos serviços odontológicos, fatores sócio-demográficos e relacionados ao estilo de vida em amostra representativa de 1.170 escolares de $7^{a}$ série do ensino municipal de Gravataí, Rio Grande do Sul, Brasil. A associação entre os desfechos e sexo; cor da pele; inserção sócio-econômica; estilo de vida sedentário; uso de álcool e tabaco; e consumo de balas, refrigerantes e chocolates foi investigada com regressão de Cox univariada. Entre os jovens estudados, $77,8 \%$ escovavam os dentes $\geq 3$ vezes/dia, 31,9\% utilizavam fio dental, 68,9\% visitavam o dentista anualmente e 50\% consultaram por motivo curativo. As meninas apresentaram maior freqüência de escovação do que os meninos. Os jovens com baixa inserção sócio-econômica, os com estilo de vida sedentário e os que experimentaram tabaco apresentaram menor uso diário de fio dental e consultas odontológicas anuais, e mais consultas curativas. O consumo de balas associou-se à menor utilização de serviços odontológicos e o de refrigerantes à maior freqüência de consultas curativas. O estilo de vida saudável associou-se com melhores hábitos de higiene bucal e utilização de serviços odontológicos.

Higiene Bucal; Serviços de Saúde Bucal; Adolescente até mesmo, suprir a falta de orientação da família sobre estilo de vida saudável. Esses e outros cuidados deveriam ser trabalhados como temas transversais em todas as disciplinas, visto que o período escolar é fundamental para abordar a promoção da saúde, contribuindo para que os jovens possam, cada vez mais, realizar escolhas saudáveis em suas vidas 6 .

As condições sócio-econômicas dos jovens apresentaram-se associadas a quase todos os desfechos investigados, com exceção à escovação pelas razões já discutidas. Esse resultado está em consonância com vários outros já publicados e aponta para o importante papel do Estado em minimizar as grandes desigualdades observadas neste país. Isso pode ser realizado com a implantação, por parte do poder público, de programas de vigilância das condições de saúde bucal e seus fatores de risco em todas as escolas públicas e também no Sistema Único de Saúde (SUS). Essa medida permitirá o monitoramento da ocorrência de agravos e do impacto das medidas adotadas, tanto para preveni-los como para evitá-los.

\section{Colaboradores}

D. R. G. C. Aerts participou de todas as etapas do estudo, desde o planejamento do mesmo até a análise dos dados e redação final do artigo. S. L. Freddo participou da coleta e análise dos dados, e redação do artigo. C. Abegg participou do planejamento do estudo e redação do artigo. R. Davoglio e P. C. Vieira participaram da coleta dos dados e redação final do artigo. L. Monteiro participou do planejamento do estudo, coleta dos dados e redação final do artigo. 


\section{Referências}

1. World Health Organization. Physical status: the use and interpretation of anthropometry. Geneva: World Health Organization; 1995. (WHO Technical Report Series, 854).

2. Palazzo LS, Béria JU, Tomasi E. Adolescentes que utilizan servicios de atención primaria: ¿Cómo viven? ¿Por qué buscan ayuda y cómo se expresan? Cad Saúde Pública 2003; 19:1655-65.

3. Hashim R, Thomson MW, Pack ARC. Smoking in adolescence as a predictor of early loss of periodontal attachment. Community Dent Oral Epidemiol 2001; 29:130-5.

4. Garcia-de-Valente MS. Saúde oral na adolescência. Adolesc Latinoam 1998; 1:170-4.

5. Claro LBL, March C, Mascarenhas TM, Castro IAB, Rosa MLG. Adolescentes e suas relações com serviços de saúde: estudo transversal em escolares de Niterói, Rio de Janeiro, Brasil. Cad Saúde Pública 2006; 22:1565-74.

6. Secretaria de Políticas de Saúde, Ministério da Saúde. A promoção da saúde no contexto escolar. Rev Saúde Pública 2002; 36:533-5.

7. Kwan S, Petersen PE, Pine CM, Borutta A. Healthpromoting schools: an opportunity for oral health promotion. Bull World Health Organ 2005; 83:67785.

8. Locker D. Concepts of oral health, disease and the quality of life. In: Slade GD, editor. Measuring oral health and quality of life. Chapel Hill: Department of Dental Ecology, School of Dentistry, University of North Carolina; 1997. p. 11-25.

9. Locker D. An introduction to behavioural science \& dentistry. London: Tavistock; 1989.

10. Honkala E. Oral health promotion with children and adolescents. In: Schou L, Blinkhorn AS, editors. Oral health promotion. Oxford/New York/Tokyo: Oxford University Press; 1993. p. 169-85.

11. Levine RS. The scientific basis of dental health education: a policy document. $4^{\text {th }}$ Ed. London: Health Education Authority; 1996.

12. Gil AM. Hábitos, costumbres, aptitudes de higiene bucal y tratamientos odontológicos realizados en la actualidad. Acta Odontol Venez 1996; 34:22-34.

13. Maltz M. Cariologia, prevenção e doença periodontal. In: Toledo D, organizador. A odontopediatria: fundamentos para a prática. São Paulo: Premier; 1996. p. 105-33.

14. Benatti RM, Trotta EA. A saúde bucal da criança e do adulto: aspectos atuais. Revista HCPA 2000; 20:37-43.

15. Trentin MS, Oppermann RV. Prevalência dos hábitos de higiene bucal interproximal e sua influência na presença de placa e sangramento gengival em um grupo de estudantes. RPO UPF 2001; 6:15-22.

16. Pinheiro RS, Aguiar FP, Sass PE, Vilela MJN. Diferenças no uso de serviços odontológicos entre os estados do Brasil: uma análise baseada em modelos hierárquicos. Cad Saúde Colet (Rio J) 2006; 14:141-8.

17. Doty HE, Weech-Maldonado R. Racial/ethnic disparities in adult preventive dental care use. J Health Care Poor Underserved 2005; 14:516-34.
18. Antunes JL, Pegoretti T, De Andrade FP, Junqueira SR, Narvai PC. Ethnic disparities in the prevalence of dental caries and restorative dental treatment in Brazilian children. Int Dent J 2003; 53:7-12.

19. Jiang H, Petersen PE, Peng B, Tai B, Bian Z. Selfassessed dental health, oral health practices, and general health behaviors in Chinese urban adolescents. Acta Odontol Scand 2005; 63:343-52.

20. Pinheiro RS, Escostegy CC. Epidemiologia e serviço de saúde. In: Medronho RA, organizador. Epidemiologia. São Paulo: Editora Atheneu; 2003. p. 361-9.

21. Sakki TK, Knuuttila MLE, Antilla SS. Lifestyle, gender and occupational status as determinants of dental health behavior. J Clin Periodontol 1998; 25:566-70.

22. Scivoletto S, Henriques Jr. SG, Andrade AG. Uso de drogas por adolescentes que buscam atendimento ambulatorial: comparação entre "crack" e outras drogas ilícitas - um estudo piloto. Rev ABP-APAL 1997; 19:7-17.

23. Swedish Council for Information on Alcohol and Other Drugs. The 1999 ESPAD report: alcohol and other drug use among students in 30 European countries. Stockholm: The Swedish Council for Information on Alcohol and other Drugs; 2000.

24 Orfaliais C, Sant'Ana CC, Araújo AJ. Diretrizes para cessação do tabagismo: abordagem de grupos especiais: crianças e adolescentes. J Bras Pneumol 2004; 30 Suppl 2:65-75.

25. National Center for Chronic Disease Prevention and Health Promotion. Physical activity and health: a report of the surgeon general. http:// www.cdc.gov/nccdphp/srg/chapcon.htm (acessado em 11/Set/2006).

26. Telama R, Yang X. Decline of physical activity from youth to young adulthood in Finland. Med Sci Sports Exerc 2000; 32:1617-22.

27. Oehlschlaeger MLK, Pinheiro RT, Horta B, Gelatti C, Sant'Ana P. Prevalências e fatores associados ao sedentarismo em adolescentes de área urbana. Rev Saúde Pública 2004; 38:157-63.

28. Raitakari OT, Leino M, Rakkonen K, Porkka KV, Taimela S, Rasanen L, et al. Clustering of risk habits in young adults: The cardiovascular risk in young Finns study. Am J Epidemiol 1995; 142:36-44.

29. Ministério da Saúde. Monitoramento da saúde do adolescente. http://portal.saude.gov.br/portal/ arquivos/pdf/projeto_escolares.pdf (acessado em 15/Out/2006)

30. World Health Organization. Child and adolescent health and development. Adolescents: the sheer numbers. http://www.who.int/child-adolescenthealth/overview/Ahd/ahdsheer.htm (acessado em 15/Jul/2005).

31. Lisbôa IC, Abegg C. Hábitos de higiene bucal e uso de serviços odontológicos de adolescentes e adultos do Município de Canoas, RS, Brasil. Epidemiol Serv Saúde 2006; 15:29-39.

32. Aguzzoli A. Hábitos de higiene bucal e utilização de serviços odontológicos em indivíduos acima de 49 anos: um estudo de base populacional em Canoas, RS, Brasil [Dissertação de Mestrado]. Canoas: Programa de Pós-graduação em Saúde Coletiva, Universidade Luterana do Brasil; 2005. 
33. Calvo MCM, Schneider Filho DA. A saúde e as doenças bucais. In: Sucupira ACSL, organizador. Pediatria em consultório. São Paulo: Editora Sarvier; 1996. p. 1095-106.

34. Harris NO, Christien AG. Primary preventive dentistry. East Norwalk: Appleton \& Longe; 1991.

35. Barros AJ, Hirakata VN. Alternatives for logistic regression in cross-sectional studies: an empirical comparison of models that directly estimate the prevalence ratio. BMC Med Res Methodol 2003; 3:21.

36. Gift HC. Current utilization patterns of oral hygiene practices: state-of-the-science-review. In: Löe H, Kleinman DV, editors. Dental plaque control measures and oral hygiene practices. Oxford: Oxford University Press; 1986. p. 39-71.

37. Brew M. Conhecimentos e hábitos dos adolescentes do ensino médio, do Município de Torres/RS, em relação à saúde bucal [Dissertação de Mestrado]. Canoas: Programa de Pós-graduação em Saúde Coletiva, Universidade Luterana do Brasil; 2002.

38. Abegg C. Desenvolvimento de comportamentos e hábitos condutores à saúde bucal. In: Bönecker M, Sheiham A, organizadores. Promovendo a saúde bucal na infância e adolescência: conhecimentos e práticas. Porto Alegre: Editora Santos; 2004. p. 97-107.

39. Flores EMTL, Drehmer TM. Conhecimento, percepções, comportamentos e representações de saúde bucal dos adolescentes de escolas públicas de dois bairros de Porto Alegre. Ciênc Saúde Coletiva 2003; 3:743-52.

40. Rodrigues HJG. Padrão de conhecimento do atleta amador de Bauru-SP, relacionado aos cuidados da saúde bucal [Dissertação de Mestrado]. Bauru: Faculdade de Odontologia de Bauru; 2005.

41. Linn EL. Teenager's attitudes, knowledge, and behaviours related to oral health. J Am Dent Assoc 1976; 5:946-51.

42. Todd J, Lader D. Adult dental health-dental 1988. London: Her Majesty's Stationery Office; 1991.

43. Hodge HG, Holloway PJ, Bell CR. Factors associated with tooth-brushing behavior in adolescents. Br Dent J 1982; 152:49-51.
44. Kandrack MA, Grant KR, Segall A. Gender differences in health related behaviour: some unanswered questions. Soc Sci Med 1991; 5:579-90.

45. Nadanovsky P, Sheihan A. The relative contribution of dental services to the changes and geographical variation in caries status of 5-and 12-year-old children in England and Wales in the 1980s. Community Dent Health 1994; (11):215-23.

46. Barros AJD, Bertoldi AD. Desigualdades na utilização e no acesso a serviços odontológicos: uma avaliação em nível nacional. Ciênc Saúde Coletiva 2002; 4:709-17.

47. Pinheiro RS, Viacava F, Travassos C, Brito AS. Gênero, morbidade, acesso e utilização de serviços de saúde no Brasil. Ciênc Saúde Coletiva 2002; 4:687-707.

48. Matos DL, Lima-Costa MF, Guerra HL, Marcenes W. Projeto Bambuí: avaliação de serviços odontológicos privados, públicos e de sindicatos. Rev Saúde Pública 2002; 36:237-43.

49. Matos DL, Lima-Costa MF, Guerra HL, Marcenes W. Projeto Bambuí: estudo de base populacional dos fatores associados com o uso regular dos serviços odontológicos em adultos. Cad Saúde Pública $2001 ; 17: 661-8$.

50. Verbrugge L. A health profile of older women with comparisons to older men. Res Aging 1984; 6:291322.

51. Kieser JA, Groeneveld HT. Inequalities in the pattern of dental delivery in South Africa. J Dent Assoc S Afr 1995; 50:327-31.

52. Gillcrist JA, Brumley DE, Blackford JU. Community socioeconomic status and children's dental health. J Am Dent Assoc 2001; 132:216-22.

53. Watt R, Sheiham A. Inequalities in oral health: a review of the evidence and recommendations for action. Br Dent J 1999; 187:6-12.

Recebido em 08/Ago/2007

Versão final reapresentada em 07/Jan/2008

Aprovado em 15/Jan/2008 